\title{
Le Moyen Âge au Nouveau Monde
}

L'enjeu culturel des Mediaeval Studies

\section{Florian Michel}

\section{CpenEdition}

\section{Journals}

Édition électronique

URL : http://journals.openedition.org/assr/21824

DOI : $10.4000 /$ assr. 21824

ISSN : $1777-5825$

Éditeur

Éditions de l'EHESS

\section{Édition imprimée}

Date de publication : 31 mars 2010

Pagination : 9-32

ISBN : 978-2-7132-2253-5

ISSN : 0335-5985

\section{Référence électronique}

Florian Michel, «Le Moyen Âge au Nouveau Monde », Archives de sciences sociales des religions [En ligne], 149 | janvier-mars 2010, mis en ligne le 05 octobre 2010, consulté le 30 avril 2019. URL : http:// journals.openedition.org/assr/21824 ; DOI : 10.4000/assr.21824 


\section{Florian Michel}

\section{Le Moyen Âge au Nouveau Monde}

L'enjeu culturel des Mediaeval Studies

Les " études médiévales " en Amérique du Nord pourraient sans doute, à première vue, constituer davantage un objet d'étonnement qu'un sujet d'étude ${ }^{1}$. Même un évêque catholique américain de la fin du XIXe siècle, que l'on aurait pu croire plutôt favorable au Moyen Âge, proclamait qu' « il ne nous servira de rien de comprendre le XIII ${ }^{e}$ siècle mieux que le XIX ${ }^{e}$ siècle. Le monde est entré dans une phase entièrement nouvelle, et le passé ne reviendra pas ${ }^{2}$. Un religieux français, invité par son ordre à fonder à Ottawa un institut d'études médiévales au commencement des années trente, remarquait, dans une perspective assez européenne, que « les Canadiens s'essaient à pénétrer eux aussi dans le Moyen Âge, qui est à la lettre pour eux de la préhistoire. Ce n'est pas le moindre obstacle » ${ }^{3}$.

Gustave Cohen (1879-1958), professeur d'histoire médiévale à la Sorbonne, que les infortunes de la Seconde Guerre mondiale conduisent à Yale en 1941, soulignait cependant combien le médiévisme était une discipline bien établie au Nouveau Monde malgré ses couleurs paradoxales et ses difficultés pratiques :

« Il semble qu'aucune civilisation ne soit plus loin de notre Moyen Âge occidental que celle toute trépidante et mécanisée des États-Unis. C'est une erreur, que j'ai depuis longtemps combattue quand j'enseignais en Sorbonne, dès la création de ma chaire en 1932, qu'ils avaient pris dans notre discipline une place d'avant-garde. (...) Une organisation universitaire magnifique, des bibliothèques abondantes, des collections de photostats, pris dans nos dépôts de manuscrits, les acquisitions de primitifs par

1. Il est vrai cependant que Beowulf, poème épique de la littérature anglo-saxonne au $\mathrm{VII}^{\mathrm{e}}$ siècle, dont les études furent relancées par J. R. Tolkien dans les années trente, et les œuvres de Geoffroy Chaucer (vers 1343-1400), sont lus aux États-Unis comme autant d'éléments du patrimoine médiéval anglais. Il est vrai, aussi, que le Québec a toujours mis en avant son identité franco-catholique.

2. Mgr John Ireland, discours tenu en 1889 à la cathédrale de Baltimore, cité dans Catholicisme, hier, aujourd'hui, demain, Le Touzey et Ané, 1948, vol. 1, p. 453, col. 1. Mgr Ireland, qui était alors archevêque de Saint Paul dans le Minnesota, est un des porte-parole de l'américanisme, condamné, peu avant le modernisme, par Léon XIII dans sa lettre Testem Benevolentiae (1899). Sur l'américanisme, voir McAvoy, 1957, 1963 ; Claude Fohlen, 1987, pp. 215-230.

3. Lettre du père M.-D. Chenu au père A. Motte, 29 décembre 1931, Paris, Archives de la Province Dominicaine de France, Fonds Chenu. 
les Musées (...) constituent un appareil formidable qui, manié et exploité par des chercheurs d'élite, peut faire progresser notre science. (...) Le De translatione studii (...) se prolonge vers l'Ouest dans le sens de la marche apparente du soleil » (Cohen, 1945 : 91-93).

Les illustrations sont alors déjà nombreuses. G. Cohen évoque ainsi la Mediaeval Academy of America, fondée en 1925 à Harvard, le Pontifical Institute of Mediaeval Studies de Toronto, fondé en 1929, et le Cloisters Museum de New York, inauguré en 1938. Il conclut sa chronique en racontant qu'au milieu de la guerre il avait pu donner un cours d'histoire médiévale à l'intérieur du Musée des Cloîtres, contemplant l'architecture médiévale en écoutant un enregistrement radiophonique des chants grégoriens de l'abbaye de Solesmes :

"Quand je prenais part en juillet août 1942 à la session d'été de Columbia University, je voulus à mon accoutumée faire avec mes étudiants une excursion médiévale. Je les conduisais aux Cloisters, au fond de l'Hudson, parmi les vieilles pierres évocatrices et authentiques venues de Saint-Guilhem-du-Désert et de sculptures transportées également de France. Loin de faire des reproches à nos hôtes, nous les louions de les avoir préservées de l'invasion, de la destruction et du pillage des modernes Barbares érudits. "

Malgré la modernité revendiquée des États-Unis, malgré quelques phénomènes de résistance, et bien avant la réception et la diffusion protéiforme de la French Theory (Cusset, 2003), les Mediaeval Studies s'implantent solidement dans le paysage universitaire nord-américain, et offrent de resituer dans une perspective plus longue les liens culturels entre la France et l'Amérique du Nord. La translatio studii, évoquée par G. Cohen, pourrait être définie comme un échange culturel rendu possible à la fois par le déplacement de matériaux d'études, originaux ou reproduits grâce à la technique moderne, par la présence outre-Atlantique de nombreux médiévistes européens, ainsi que par la venue en Europe de nombreux chercheurs américains. Elle accompagne la passation de pouvoirs entre les deux continents qui caractérise la première moitié du "siècle américain ». L'empire américain, nouvelle Rome au temps de la chute d'une nouvelle Constantinople, a cherché à conserver sur son sol et à entretenir l'héritage culturel du Moyen Âge européen.

\section{Les balbutiements des Mediaeval Studies (années 1920)}

Les Mediaeval Studies en Amérique du Nord ont des racines académiques. On peut les dater des lendemains de la Première Guerre mondiale, durant laquelle des milliers de jeunes Américains, de tous les milieux sociaux, entrèrent en contact avec l'Europe et son passé médiéval. Avant la première guerre, le médiévisme en Amérique du Nord, plutôt marqué par une vision romantique du Moyen Âge et orienté essentiellement vers le Moyen Âge anglo-saxon en ses versants littéraire et juridique, repose sur les efforts dispersés d' " érudits solitaires » (Gentry, Kleinheinz, 1982). Deux institutions universitaires très différentes, l'Université d'Harvard et 
la Catholic University of America de Washington, témoignent des premiers pas des Mediaeval Studies au mitan des années vingt.

Le premier jalon est le mois de décembre 1925, lorsque naît, à Boston, sur le campus de l'Université d'Harvard, la Mediaeval Academy of America, fondée et dirigée par Edward Rand, George Coffman, Ralph Cram et Charles Homer Haskins (1870-1937). Ce dernier est l'âme de la fondation : c'est un ancien élève de l'École des chartes; il passe six ans en Europe, au tournant du siècle, entre Paris, Londres et Palerme, se spécialise dans l'histoire des Normands et ancre ses travaux dans une approche à la fois administrative et politique du Moyen Âge ; il est nommé professeur à Harvard en 1912 et est l'un des trois proches conseillers du Président Wilson à la conférence de Versailles en 1919 (Haskins, 1933 ; Cantor, 1993 : 254 ssq.) L’Académie Médiévale, la première de ce type aux États-Unis, lance la publication, en janvier 1926, d'une nouvelle revue intitulée Speculum, a Journal of Mediaeval Studies. E. Rand en signe le manifeste, publié dans les premières pages de la première livraison de Speculum. Il revendique "l'héritage médiéval de l'Amérique » : "La formation en Amérique d'une Académie Médiévale est un signe des temps encourageant. La conception du Moyen Âge comme une période d'ignorance crasse, de mauvais goût et de fanatisme aveugle ne trouve plus beaucoup de défenseurs. » Le titre Speculum, qui évoque « les miroirs multiples où les gens du Moyen Âge aimaient à se regarder », propose de donner un reflet médiéval au sein du monde moderne. Le projet est "d'étudier tout le Moyen Âge ", avec un accent particulier placé sur "le Moyen Âge latin, qui est le centre de nos intérêts, mais n'en est pas la circonférence. »

Un article, paru également dans le premier numéro de Speculum, donne de saisir la genèse de cette "Académie Médiévale »(Coffman, 1926 : 5-18). L'impulsion date de 1920 ; elle a été donnée par le Pr. John Manly, de la Modern Language Association, qui constatait l'absence d'organisation des «Mediaeval Studies dans ce pays ». En 1921, est lancé un programme de Mediaeval Latin Studies, qui peu à peu se solidifie en Mediaeval Academy. Le médiévisme, à Harvard, trouve donc son origine dans les études linguistiques, puisque la faculté des langues, désireuse d'approfondir le champ du latin médiéval, en suggère l'organisation. L'accent est alors mis sur la littérature et la latinité médiévale. La fondation de la Mediaeval Academy est un moment charnière dans l'histoire des études médiévales en Amérique et fait de Cambridge le haut lieu du médiévisme outre-Atlantique (Gentry, Kleinheinz, 1982 : 13-19).

Le second jalon est posé lors de la naissance, en janvier 1926, de l'American Catholic Philosophical Association ${ }^{4}$, qui lance un an plus tard à Washington, aux presses de la Catholic University of America, une revue intitulée The New Scholasticism:

4. Pour l'American Catholic Philosophical Association, voir les Proceedings of the First Annual Meeting, tenu à la Catholic University of America le 5 janvier 1926, organisé par Mgr Thomas Shahan et le père James Ryan. 
"Nous avons la conviction, disait Mgr Edward Pace lors du discours inaugural de l'association, que les idées de base de la scolastique sont des vérités vivantes, assez solides pour porter l'entièreté du savoir et assez flexibles pour autoriser tous les ajouts des faits connus $»^{5}$.

La perspective de The New Scholasticism est celle d'un retour à la philosophie médiévale, et au renouveau thomiste en particulier tel qu'il s'est esquissé en France et en Belgique, notamment, sous les auspices du Saint-Siège, dans la postérité tardive de l'encyclique Aeterni Patris (1879):

«Depuis trop longtemps nous sommes considérés comme un peuple et un clergé de grande énergie, et de grand zèle, qui construisons, organisons et défendons toutes sortes d'institutions, mais étrangers à toute poursuite des biens intellectuels. Le moment pour de telles conceptions est révolu. (...) L'association ouvre une nouvelle ère dans la vie catholique de notre pays ${ }^{6}$.

Le premier numéro de la revue s'ouvre par un hommage au cardinal Mercier de Louvain qui venait de disparaître, et par la communication d'Étienne Gilson au Congrès International de Philosophie qui s'était tenu à Boston quelques mois plus tôt ${ }^{7}$.

Un ensemble de signes plus diffus confirme la lente montée en puissance de l'intérêt pour le Moyen Âge en Amérique tout au long des années vingt. À partir de 1926, James F. Willard, vice-président de l'Académie Médiévale et professeur d'histoire médiévale à l'Université du Colorado, publie ainsi une brochure intitulée Progress of Mediaeval Studies in the United States of America, où l'auteur énumère les travaux entrepris aux États-Unis, ainsi que les noms de tous les historiens des États-Unis qui s'y occupent du Moyen Âge : ils sont soixante en 1923, dépassent les trois cents en 1926 pour atteindre huit cents en 1940 $\left(\right.$ Willard, 1926) ${ }^{8}$. Le Moyen Âge historique ne peine donc pas à s'introduire dans la vie académique américaine, même si, hormis à Harvard avec Charles H. Haskins qui envoie dans les universités américaines nombre de ses étudiants (Cantor, 1993 : 254), l'effort de structuration de la discipline est encore à réaliser.

Cependant, le Moyen Âge intellectuel peine davantage à convaincre les milieux non catholiques. En 1926, à New York, John Zybura publie une enquête où sont rapportés les témoignages des philosophes américains les plus en vue sur la valeur de la philosophie médiévale et sur ses rapports avec la pensée moderne.

5. Edward Pace, "Inaugural Address ", in Proceedings of the First Annual Meeting, janvier 1926, p. 16.

6. Id., p. 18.

7. The New Scholasticism, janvier 1927, Catholic University Press: Maurice de Wulf, "Cardinal Mercier : philosopher ", Étienne Gilson, "Le rôle de la philosophie dans l'Histoire de la civilisation".

8. L'ouvrage est ensuite publié annuellement en supplément de Speculum sous le titre Progress of Mediaeval and Renaissance Studies par James Willard jusqu'en 1937 et par S. Harrison Thomson au-delà. 
Les remarques sont parfois très critiques. Ralph Barton Perry, professeur de philosophie à Harvard, explique ainsi que « la présente dérive vers le réalisme pourrait rallumer l'intérêt envers la philosophie scolastique ", mais il ajoute que le poids de l'autorité et la pesanteur du dogme anéantissent l'effort scolastique : «l'esprit de la philosophie moderne séculière est radicalement critique : il ne tolère pas la fermeture d'une question, mais reconsidère toutes croyances "; "l'issue du philosopher scolastique est déterminée à l'avance " : c'est un empty show ${ }^{9}$. John Dewey, le leader pragmatiste de l'École de Chicago, reconnaît également qu' " il y a, pour la scolastique, un intérêt croissant, lié pour partie au renouveau des théories réalistes de la connaissance, et pour partie à la multiplication des études sur Aristote et l'intérêt corollaire pour les penseurs médiévaux »; mais l'ensemble des philosophes américains demeurent étranger au monde scolastique. Les causes de cette indifférence sont nombreuses, explique Dewey: «Les philosophes non scolastiques ont été élevés dans la tradition protestante et ont - plus ou moins inconsciemment - identifié la scolastique avec les dogmes théologiques qu'ils n'acceptent pas » ${ }^{10}$. Pour le philosophe Santayana, "la fixité et la clarté du vocabulaire scolastique sont une consolation du Babel des termes figuratifs et des catégories perverses qui rendent confuse la philosophie moderne ", mais la scolastique ne peut pas susciter l'intérêt des contemporains : elle est «trop lointaine ${ }^{11}$.

Dès les années vingt, on repère donc en Amérique du Nord un réel attrait pour le médiévisme, et notamment pour le Moyen Âge intellectuel. Mais il faut noter une forte résistance selon la période considérée. Le Moyen Âge historique est consensuel et se trouve intégré pleinement dans le champ universitaire et culturel. L'année 1925 est aussi le moment où une importante donation financière du philanthrope John D. Rockfeller (1874-1960) permet la création du Cloisters Museum (Rorimer, 1938) ${ }^{12}$. Mais, alors même qu'il commence à exister un Moyen Âge à destination du grand public américain, qui peut ainsi déambuler dans quelques cloîtres français, le Moyen Âge philosophique et métaphysique devient, quant à lui, l'objet d'une lutte riche de potentialités dialectiques (Michel, 2007 : 33-56). Ce n'est pas le lieu de montrer ici comment la philosophie dite médiévale et la philosophie dite américaine sont devenues les deux forces conflictuelles de la vie philosophique aux États-Unis au lendemain de la Seconde Guerre mondiale : "Les deux courants de pensée qui paraissent aujourd'hui prévalents aux États-Unis, écrit Maritain, sont le courant pragmatiste et le courant, comme on dit, "néo-thomiste" (Maritain, 1944 : 271-300). Il suffit de souligner la latence du conflit et la variété du Moyen Âge considéré : la Catholic University

\footnotetext{
9. Ralph Barton Perry, in Zybura, 1926, pp. 3-4.

10. John Dewey, in Zybura, 1926, pp. 29-31.

11. George Santayana, in Zybura, 1926, pp. 74-76.

12. James Rorimer, 1938, (rééd. 1963). La première édition contient dans sa préface une brève notice historique sur la provenance des collections du musée.
} 
of America décide d'investir le terrain de la philosophie médiévale ; J. Dewey reconnaît, pour sa part, que « l'éducation protestante » prévenait contre la philosophie scolastique ; l'Université d'Harvard s'engage, quant à elle, dans l'étude du Moyen Âge politique et institutionnel.

\section{Les Mediaeval Studies : les trois premières fondations}

Les universités catholiques sont les premières à comprendre l'importance qu'il y aurait à structurer fortement les études médiévales. Trois institutions, deux au Canada et une aux États-Unis, germent ainsi dans l'entre-deux-guerres. La première, fondée par le médiéviste français Étienne Gilson (1884-1978), naît à Toronto en 1929 ; reconnue par la Congrégation romaine des Séminaires et Universités, elle devient le Pontifical Institute of Mediaeval Studies en 1939. La seconde institution, fondée par les dominicains de la Province du Canada, prend forme, en 1930, au sein du couvent d'Ottawa, avant d'être déplacée, en 1942, à l'Université de Montréal. La troisième surgit sur le campus de l'Université de Notre Dame, au nord de l'Indiana, dans la seconde moitié des années trente, mais ne prend son essor qu'au lendemain de la Seconde Guerre mondiale.

L'histoire du Pontifical Institute of Mediaeval Studies (PIMS) s'inscrit dans un projet d'études du Moyen Âge philosophique et théologique. Né dans le cadre de Saint-Michael's College, rattaché à l'Université de Toronto, l'institut est tenu par la congrégation de Saint-Basile.

En 1926, dans le cadre d'un échange universitaire, Étienne Gilson, alors professeur de philosophie à la Sorbonne, est nommé visiting professor pour un semestre à l'Université d'Harvard, qui lui propose, en 1927, une chaire professorale " pour renforcer le département de philosophie dans ce qui est maintenant son maillon le plus faible, à savoir l'histoire " ${ }^{13}$. Au même moment, des religieux de la congrégation de Saint-Basile donnent carte blanche à Gilson pour fonder à Toronto un laboratoire d'études médiévales. Gilson accepte la proposition basilienne, renonce à l'offre d'Harvard et montre que son Moyen Âge ne correspond guère à celui d'Harvard: "Quant à l'histoire de la philosophie, ici [à Harvard] on n'en voit pas l'usage, écrit Gilson en 1926. Perry est assez troublé. Il pense que trop étudier les systèmes des autres empêche les jeunes de trouver le leur. C'est comme si visiter les musées et étudier les maîtres empêchaient de devenir peintre " ${ }^{14}$. "Je sais qu'Harvard est Harvard, mais c'est pour moi une occasion unique de réaliser mon idéal des études médiévales " ${ }^{15}$. L’Institut de Toronto est officiellement ouvert à la rentrée de 1929. Un article avait paru

13. Lettre de William Hocking au Président Lawrence Lowell, 21 décembre 1926, Archives Harvard.

14. Lettre d'É. Gilson à son épouse Thérèse, 6 octobre 1926, citée par L. Shook, op. cit., 1984, p. 150 et retraduite à partir de l'anglais.

15. Lettre d'É. Gilson au Professeur Wood, 28 janvier 1928, Archives Harvard. 
quelques mois plus tôt pour en présenter les fins : " recouvrer une civilisation et une culture, qui ont été, pour une large part, perdues » ${ }^{16}$.

Étienne Gilson, soutenu par les Pères basiliens, parvient à organiser en une décennie un actif centre de recherches sur le Moyen Âge : il forme une équipe de professeurs compétents et rassemble les instruments de recherche adéquats. Cette croissance, remarquable en si peu d'années, est la fierté de Gilson. Il s'en ouvre à Ralph Barton Perry, son collègue d'Harvard: "Mon institut d'études médiévales n'est plus un vague rêve, écrit-il. J'ai dix professeurs, chacun d'eux spécialisé dans un champ particulier de la civilisation médiévale. (...) Je suis un homme heureux, plus qu'heureux : contenté ${ }^{17}$. Elle suscite une admiration jalouse à Harvard : "Félicitations pour la croissance et la fécondité de l'Institut ! lui répond Perry. Vous avez réussi à trouver dix hommes en philosophie médiévale, tandis que jusqu'à aujourd'hui nous n'en avons pas trouvé un seul. Peut-être un jour vous en trouverez un pour nous ${ }^{18}$.

Le champ d'études de l'Institut de Toronto est assez large. L'Institut avait d'abord pour fin de permettre une meilleure compréhension des grands docteurs médiévaux en les resituant dans leur époque. Dans un premier temps, Gilson avait demandé à Rome une reconnaissance canonique pour une faculté de philosophie et de théologie. Il s'agissait de mettre l'histoire médiévale au service de ces deux disciplines. Assez vite cependant, l'ensemble de la civilisation médiévale latine, de la musique à la littérature, de la liturgie à l'architecture, est étudié et enseigné par les professeurs du PIMS, même si philosophie et théologie y demeurent les disciplines reines.

À la veille de la Seconde Guerre mondiale, en 1939, l'Institut se dote de la revue Mediaeval Studies, qui assure la diffusion des travaux de l'Institut et édite, encore aujourd'hui, de nombreux manuscrits médiévaux. "La revue fut la clé de voûte qui assura la solidité de l'ensemble de l'entreprise ; c'était un élément indispensable pour attirer étudiants et professeurs »(Owens, 1988 : XI-XVI).

L'Institut prospère jusqu'au Concile de Vatican II. On trouve un témoignage de «l'âge d'or» du PIMS sous la plume du médiéviste belge Fernand van Steenberghen :

"L'organisation matérielle de l'Institut est remarquablement bien adaptée à son but, qui est de former une élite de chercheurs parfaitement exercés aux méthodes scientifiques dans le domaine de l'histoire médiévale. (...) Il s'agit de former une élite de travailleurs, dont chacun doit être suivi de près par ses maîtres. La bibliothèque admirablement outillée pour les études médiévales, compte quelque 20000 volumes, 20 incunables, 400 manuscrits reproduits photographiquement et une collection de

16. É. Gilson, "Mediaevalism in Toronto ", The Commonweal, New York, $1^{\text {er }}$ mai 1929, pp. 738-740.

17. Lettre d'É. Gilson à Ralph B. Perry, 2 novembre 1935, Archives Harvard.

18. Lettre de Ralph B. Perry à É. Gilson, 14 novembre 1935, Archives Harvard. 
microfilms qui contient déjà environ 120000 pages de manuscrits ou de livres rares » (1951: 411-412).

L'Institut de Gilson suscite l'admiration des médiévistes nord-américains : «Toronto est le lieu où Dieu envoie tous les bons médiévistes quand ils meurent, et où il envoie la plupart des meilleurs médiévistes longtemps avant leur mort " (Berkhout, 1982 : 105), et engendre en écho d'autres fondations, en Europe, à Louvain par exemple (Van Steenberghen, 1994 : 17-21), ou en Amérique du Nord. Élèves et professeurs du PIMS essaiment à travers le continent, vers les universités catholiques ou séculières. Son mode d'organisation des études sert aussi de modèle : les «études médiévales " ne sont pas une unité au sein de la faculté d'histoire, comme souvent en France, mais un « institut » pluridisciplinaire, qui étudie les divers aspects de la civilisation médiévale.

L'Institut d'Études Médiévales d'Ottawa est l'équivalent francophone de l'Institut de Toronto. Fondé, en 1930, au sein du couvent dominicain d'Ottawa, porté sur les fonts baptismaux par É. Gilson et le P. Marie-Dominique Chenu, l'Institut se déplace, en 1942, à l'Université de Montréal où il est rattaché à la Faculté de Philosophie. Sans surprise, l'Institut, qui prend alors le nom d'Albert le Grand, choisit pour spécialité le Moyen Âge philosophique et dominicain.

De 1930 à 1942, l'Institut, dont les professeurs appartiennent pour la plupart à l'Ordre de saint Dominique, s'adresse d'abord aux étudiants dominicains du Nouveau Monde. Confiné dans les bâtiments du couvent, le rayonnement de l'Institut dans la capitale canadienne est limité, même si sa visibilité internationale est assurée par une collection de co-publications avec l'éditeur Vrin, à Paris, qui publie ainsi une dizaine de titres entre 1932 et $1940^{19}$.

De 1942 à la fin des années quatre-vingts, au terme desquelles il disparaît au fil de diverses restructurations universitaires, l'Institut jouit d'une belle notoriété. Il est alors une faculté complète, dirigée par un conseil pédagogique propre, pourvu d'une organisation scolaire autonome ayant son groupe de professeurs et d'élèves. Il attire quelques professeurs de renom, comme Henri-Irénée Marrou, Paul Vignaux ou Raymond Klibansky. L'Institut connaît à Montréal ses grandes heures :

«À Montréal même, et à l'intérieur de l'Université, l'influence de l'Institut a été accrue, note son directeur, en 1948. L'ampleur du programme offert à nos élèves en même temps que la pratique rigoureuse des méthodes historiques amènent des relations inévitables avec les autres facultés qui peuvent trouver chez nous un complément nécessaire de leur propre enseignement, au plan tant des objets que des techniques ${ }^{20}$.

19. Le premier volume de la "Collection de l'Institut d'Études Médiévales d'OttawaMontréal " s'intitule Études d'histoire littéraire et doctrinale $d u \mathrm{XIII}^{e}$ siècle, première série, Ottawa, IEM et Paris, Vrin, 1932.

20. RP. Régis, "Mémoire sur l'Institut d'Études Médiévales présenté au P. Emmanuel Suarez, Maître Général de l'Ordre des Frères Prêcheurs ", 31 mars 1948, p. 13, Montréal, Archives de la Province Dominicaine du Canada. 
Les publications sont nombreuses. La " conférence Albert-le-Grand ", chaque automne, est un événement de qualité. La translation à Montréal est plus qu'un simple déplacement géographique. L'Institut entre alors dans l'aire gravitationnelle complexe et métamorphique d'une grande université. Pierre Boglioni, ancien élève et ancien directeur, commente ainsi la venue à Montréal de l'Institut :

"L'Institut d'Études médiévales allait aussi être rattaché à l'Université de Montréal, par le biais de la Faculté de Philosophie, en fournissant à cette faculté, dès le début, une partie de son personnel. (...) Il correspondait en somme au modèle de la Graduate School américaine ou, avec des moyens et un personnel plus réduits, au modèle du Pontifical Institute of Mediaeval Studies " (Boglioni, 2001 : 195-196).

L'intégration au sein du monde académique montréalais ira croissante. L'Université de Montréal, catholique jusqu'alors, est sécularisée à la fin des années soixante. L'étiolement du thomisme induit dans les années soixante-dix une laïcisation de l'étude du Moyen Âge. Les études médiévales à Montréal témoignent de la révolution culturelle que connut alors le Québec. En quatre décennies, on passe de l'étude de la philosophie au Moyen Âge à l'étude de la religion populaire médiévale, puis à celle de la marginalité, de la grossièreté ou de l'érotisme au Moyen Âge, et enfin à l'étude de la religion populaire au Québec (Panaccio, 1998 : 145-162).

«Ce que Saint Michael's College a fait, Notre Dame peut le faire " ${ }^{21}$. La fondation de l'Institut d'Études médiévales de l'Université de Notre Dame, Indiana, puise à la même source que les instituts de Toronto et Ottawa. À Notre Dame, le projet de l'Institut, le premier de ce type aux États-Unis, date de l'entre-deuxguerres. Le P. Philip Moore (1900-1969), de la Congrégation de Sainte-Croix, ancien chartiste, ancien élève de Gilson à Paris, et principal architecte du nouvel institut, en retrace ainsi la généalogie : "The Mediaeval Institute a été formellement fondé en 1946, mais il provenait d'un premier programme en études médiévales qui remontait à 1933 » (1960). L'absence de personnel qualifié et l'insuffisance des fonds grèvent l'initiative lancée par Gilson :

«Le problème de Notre Dame est beaucoup plus difficile à résoudre, écrivait Gilson en 1936. J'ai rencontré il y a quelques années un jeune père américain de Sainte-Croix, qui étudiait alors à l'École des chartes, le P. Philippe Moore. Comme il se demandait ce qu'il ferait (et incidemment ce qu'on lui demanderait de faire) après l'obtention de son diplôme, je lui avais suggéré l'organisation d'un Institut américain, plus ou moins sur le modèle de celui de Toronto et dirigé par Notre Dame. (...) L'an passé, alors que je donnais des conférences là bas, (...) je leur ai laissé une série de recommandations » ${ }^{22}$.

À partir du milieu des années trente, l'Université de Notre Dame n'offre qu'un " programme de cours en études médiévales ", qui, à peine lancé, disparaît après avoir laissé dans les bibliothèques les publications de quelques volumes en

21. Lettre du père Moore au père James Burns, 8 juillet 1931, Province Archives Center, University of Notre Dame.

22. Lettre d'É. Gilson à Anton Pegis, 10 juillet 1936, Toronto, Saint Michael's College Archives. 
Mediaeval Studies (Moore, 1936 ; Corbett, Moore, 1938). En 1946, le projet d'Institut d'Études médiévales est relancé grâce aux efforts du P. Moore, et grâce à la venue du P. Gerald Phelan, ancien président du PIMS de Toronto, recommandé par Gilson.

"J'ai écrit au P. Moore, écrivait Gilson au P. Phelan, et je peux vous assurer qu'il sait maintenant combien j'espère que vous puissiez aller à Notre Dame afin de poursuivre, en un nouveau lieu, le bon travail que vous avez si bien réalisé à Toronto. (...) Bien sûr, il y aura des difficultés pratiques, mais la nature locale de ces difficultés est ce qui donnera au nouvel Institut son individualité et son caractère une fois qu'elles seront surmontées. Vous ne pouvez pas imaginer combien je désire avec ferveur que le nouvel Institut soit un succès pour le bien même du PIMS. (...) Il n'y a pas l'ombre d'une rivalité. Je suis chaudement en faveur de l'émulation » ${ }^{23}$.

À Notre Dame, de 1946 à 1952, le P. Phelan dirige les premières années du jeune institut, qui poursuit ensuite sa route sous la direction du P. Astrik Gabriel, prémontré hongrois. L'Institut quitte le strict terrain de la philosophie médiévale et se spécialise désormais dans l'histoire des écoles et de l'éducation au Moyen Âge. Il publie deux collections : "The Publication in Mediaeval Studies ", dont le premier volume était celui du P. Moore, en 1936; et "Texts and Studies in the History of Mediaeval Education », dont le premier numéro sort en 1953 (John, 1953). Il noue un échange avec la Bibliotheca Ambrosiana de Milan et fait reproduire presque l'intégralité des douze mille manuscrits de Milan ${ }^{24}$.

Sans avoir jamais ni l'ampleur ni la vigueur des instituts de Toronto et Montréal, la fondation de Notre Dame traverse les ans et propose des cours dans tous les champs de la civilisation médiévale latine : théologie et philosophie bien sûr, mais aussi paléographie, histoire de l'art, histoire de la liturgie, du droit canon, littérature médiévale et anglais médiéval. Les années soixante-soixantedix, sans être un moment de rupture, comme ce fut le cas à Montréal, voient un processus de sécularisation plus subtil, par le biais d'un léger élargissement thématique : d'une histoire religieuse de la théologie et de la philosophie médiévale, on passe à une histoire intellectuelle des lieux et des institutions du savoir au Moyen Âge.

Les trois institutions sont complémentaires sur le plan de la géographie culturelle et linguistique nord-américaine : au PIMS canadien et anglophone s'ajoutent, au fil de leur création, deux instituts analogues, l'un pour le Canada francophone, l'autre pour les États-Unis. L'ombre de Gilson est partout : "J'ai passé quelques jours à Notre Dame, où je leur ai apporté de l'aide pour organiser un Institut d'Études médiévales. Cela deviendrait-il une nouvelle manie ? ", se demande l'historien de la philosophie ${ }^{25}$. À maints égards, Gilson, qui permet l'enracinement du

23. Lettre d'É. Gilson au père G. Phelan, 9 août 1946, Toronto, Saint Michael's College Archives.

24. Pour une notice de l'" Ambrosiana Microfilm and Photographic Collection ", voir Berkhout, 1982, p. 104.

25. Lettre d'É. Gilson à Ralph Barton Perry, 25 novembre 1934, Archives Harvard. 
Moyen Âge en son versant philosophique et théologique, apparaît comme le principal vecteur de la structuration du médiévisme en Amérique du Nord ${ }^{26}$. Ces trois institutions sont les premières des vingt centres de recherche et des quatre-vingts programmes en études médiévales répertoriés au seuil des années quatre-vingts, même si à cette date les « Mediaeval Studies » ont fortement changé dans leurs méthodes comme dans leurs finalités (Berkhout, 1982: 97-120).

\section{Les vecteurs de l'échange culturel}

L'introduction du médiévisme en Amérique du Nord s'est appuyée sur deux mouvements complémentaires. Il y a, d'une part, l'importation de matériaux médiévaux, qui permet la nécessaire acclimatation du médiévisme dans les bibliothèques américaines; il y a aussi un échange transatlantique qui conduit quelques médiévistes européens à enseigner en Amérique du Nord et qui donne à de jeunes et souvent brillants étudiants américains l'occasion d'étudier le Moyen Âge en Europe.

Non sans humour, on a fait remarquer que l'architecture médiévale avait moins bien traversé l'océan que les manuscrits (Berkhout, 1982 : 98). Le Cloisters Museum de New York est l'exception notable, puisque l'on trouve, sur les bords de l'Hudson, cinq cloîtres, ainsi que des tombes, des porches, des autels, rassemblés un peu avant la Première Guerre mondiale par le collectionneur américain George Grey Barnard. Il est vrai, cependant, que le Cloisters est plutôt unique en son genre et que les manuscrits ont davantage voyagé. On a peu idée de l'ampleur de ce type d'échange transatlantique qui recouvre en fait deux réalités distinctes, puisque ce sont à la fois les originaux et les reproductions d'originaux qui sont à considérer.

Entre 1925 et 1935, deux auteurs, Seymour de Ricci et W. Wilson (19351940), s'attachèrent à répertorier tous les manuscrits du Moyen Âge et de la Renaissance présents en Amérique du Nord. Leur projet, resté sans équivalent du fait de l'ampleur de la tâche, aboutit à la publication, entre 1935 et 1940, de plus de deux milles pages en trois volumes qui cartographient la répartition du matériau médiéval en Amérique du Nord. Il n'est guère possible de donner un nombre, même approximatif, de tous les manuscrits médiévaux alors en Amérique du Nord. Pour 1940, les deux auteurs indiquent environ cinq propriétaires de manuscrits, parmi lesquels des universités, des bibliothèques publiques, des musées, des communautés religieuses et des collectionneurs privés. Certains propriétaires n'ont que quelques manuscrits. La bibliothèque de Columbia University en possède alors plus de cinquante ; la New York Public Library en possède plus de cent cinquante; la George Plimpton Library, intégrée plus tard à la

26. Voir aussi Cantor, 1993, p. 331, selon lequel «il n’y a pas de médiéviste plus influent que Gilson » aux États-Unis dans les années cinquante. 
bibliothèque de Columbia, possède plus de trois cents pièces. La Pierpont Morgan Library, à New York, est une des plus riches en mediaevalia: sans compter les divers autographes et papyrus, on y trouve environ huit cents bibles, lectionnaires, rituels, livres d'heures, psautiers ou manuscrits médiévaux. La provenance de ces manuscrits est diverse : achat aux enchères à Paris, Rome, ou Istanbul, rachat de bibliothèques complètes de lords anglais désargentés. L'Université de Chicago donne un exemple de l'ampleur du transfert culturel en jeu : en 1891, elle achète ainsi trois cent mille volumes, dont de nombreux manuscrits, sur le marché de Berlin. En 1935, elle possède plus de mille six cents manuscrits anciens. L'Art Institute of Chicago commence en 1915 sa collection d'enluminures, qui n'aura de cesse de s'enrichir. L'introduction du dernier tome de l'entreprise "Seymour de Ricci - Wilson » signalait l'importance du phénomène et invitait les chercheurs américains à exploiter et publier ces originaux :

"Les chercheurs américains ont été les principaux bénéficiaires du processus migratoire des matériaux de recherche ; ce processus semble loin d'avoir atteint son sommet. (...) Un nombre vraiment énorme de manuscrits, livres, et œuvres d'art ont été rendus accessibles pour les chercheurs américains; et cela a enrichi la vie intellectuelle des États-Unis et du Canada. Une telle situation cependant confère une lourde obligation. »

La Seconde Guerre mondiale marque une césure dans l'acquisition du matériau médiéval. À partir de 1940, et même si le mouvement avait été initié préalablement avec le procédé des photostats, les institutions ne privilégient plus l'acquisition des originaux. Les pays d'Europe deviennent plus vigilants quant à la conservation de leur patrimoine. On envisage alors la reproduction des manuscrits médiévaux sous forme de microfilms. Au seuil des années quatre-vingts, on évalue ainsi à un demi-million le nombre de manuscrits accessibles sous ce format aux États-Unis (Berkhout, 1982 : 101). Les manuscrits médiévaux de la Bibliotheca Apostolica Vaticana, de la Bibliotheca Ambrosiana de Milan, des bibliothèques d'Autriche, et d'Angleterre ont été dans leur majeure partie reproduits, respectivement, à Saint Louis University au sein de la Vatican Film Library, à l'Université de Notre Dame au sein de l'Institute of Mediaeval Studies, à Saint John's University à Collegeville dans le Minnesota et à la Library of Congress.

Outre les manuscrits, nombreux sont les médiévistes à partir, pour un semestre ou quelques années, en Amérique du Nord. Gilson offre sans aucun doute l'exemple le plus emblématique du médiéviste européen «à cheval » sur l'Atlantique : pendant cinquante ans, son année universitaire est divisée à parts presque égales entre l'Amérique du Nord et la France (Shook, 1984).

Les médiévistes français entrent, en effet, rarement dans la catégorie des émigrés. On compte certes quelques exilés du fait de la guerre. Gustave Cohen insistait à juste titre sur l'importance de l'apport des réfugiés français sur le plan des études médiévales : il aurait pu ajouter les émigrés allemands, puisque Ernst Kantorowicz (1895-1963) arriva à Berkeley en 1939. "C'est pour y enseigner le Moyen Âge que, dépossédé de ma chaire de Sorbonne, je fus appelé comme 
visiting-professor à l'université Yale ", écrit-il (1945: 91-93). Cohen évoque maintes figures du médiévisme européen exilées comme lui aux États-Unis ${ }^{27}$ : Henri Focillon, premier président de l'École Libre des Hautes Études de New York, qui donnait des cours sur l'art médiéval, Jacques Maritain, qui enseignait la philosophie scolastique, Alexandre Koyré et Paul Vignaux qui « faisaient au Moyen Âge une part royale ». "Ainsi la tradition du vieux collège que Robert de Sorbon fonda en 1259 aux bords de la Seine se continuait au bord du Connecticut » (Cohen, 1942: 73). De cette présence des médiévistes français en Amérique, Alain de Libera soulignera l'importance structurelle :

"Étienne Gilson a eu deux carrières, française et canadienne, une influence générale diffuse, disons "culturelle" en France, une autre plus profonde, plus pédagogique outre-Atlantique, deux œuvres aussi, l'une en français, l'autre en anglais. Ce double enracinement n'était pas d'ailleurs propre à Gilson : Alexandre Koyré (...) a lui aussi exercé en Amérique du Nord, comme plus tard Vignaux à Montréal. Ce passage du médiévisme français dans le monde anglo-saxon n'est pas un épisode : il fait partie de son histoire foncière" (Libera, $1991:$ 39-40).

Pour le père Chenu, Gilson ou Maritain, les liens avec l'Amérique du Nord débordent la Seconde Guerre mondiale en amont et en aval. Le père Chenu vient ainsi enseigner cinq semestres à Ottawa et Montréal entre 1930 et 1940 ; il y retourne au moment du Concile. Gilson note sur un carnet personnel le nombre de voyages outre-Atlantique qu'il réalise entre 1926 et 1953 : un par an à l'exception de la période de la guerre, soit quelque cinquante traversées de l'océan ${ }^{28}$. "J'allais enseigner de ville en ville, écrit-il, comme un wandering scholar du temps passé » ${ }^{29}$. La figure du missionnaire affleure parfois : "J'ai travaillé, pendant plus de vingt ans, à transmettre à l'Amérique une part de cette civilisation chrétienne du Moyen Âge que, par Alcuin, la France hérita jadis de Rome " ${ }^{30}$. Le parallélisme entre Gilson et Alcuin d'York est du reste souvent noté :

"M. Gilson compare sa vocation de missionnaire intellectuel à travers le monde occidental et américain à celle des "maitres itinérants du Moyen Âge". Je suis prêt à pousser l'indiscrétion jusqu'à identifier "l'itinérant" au moine Alcuin venu d'York en Francie au temps de Charlemagne. Cette fois York est Paris, et la Francie, le Canada. Grâce au "nouvel Alcuin", les arbres du Paradis avec leurs fruits continuent à croître ici ${ }^{31}$.

Quant à Maritain, entre son premier voyage, en 1933 et son dernier voyage, en 1966, il fait une quinzaine d'allers-retours. Après la guerre, il entend bien

27. Pour une histoire de l'École Libre des Hautes Études, voir Loyer, 2005.

28. Pages reproduites dans F. Michel, Un réseau d'intellectuels européens en Amérique du Nord. Diffusion, réception et américanisation de la pensée catholique. Années 1920années 1960, Thèse dirigée par C. Langlois, EPHE, 2006, pp. 659-660.

29. É. Gilson, "L'Esprit de Chrétienté ", La Vie Intellectuelle, février 1945, pp. 18-19.

30. Lettre d'É. Gilson à Albert Béguin, 11 mars 1951, Journal à plusieurs voix, Paris, 1951, p. 596.

31. Benoît Lacroix, "Un centre de savoir et de culture ", Relations, Montréal, 1947, p. 317. 
demeurer un philosophe atlantique. À un ami canadien il écrit ainsi en 1945 : "C'est un au revoir que je vous dis. Il est clair que je ne veux pas rompre les liens qui m'attachent au Canada et aux États-Unis " ${ }^{32}$. On retrouve le même vœu dans une lettre à François Mauriac au printemps 1948 : " Je vais reprendre, avec ma vocation de philosophe, mon métier de professeur errant d'un bord à l'autre de l'océan, ce qui est sans doute plus conforme à ma destinée que les stables cathèdres du Collège de France ${ }^{33}$.

La dernière modalité de ce transfert concerne la venue d'Américains en Europe dans le but d'étudier le Moyen Âge in situ. L'exhaustivité et la quantification sont difficiles. Mais le processus, en revanche, est vérifiable par de nombreux exemples; qualitativement, il est très significatif.

Pour l'entre-deux-guerres, on note ainsi un flux régulier d'étudiants américains, constituant souvent l'élite future de l'institution qui les envoie en Europe. Les parcours d'études de nombreux doyens, professeurs d'université et prélats américains dessinent des trajectoires parallèles et désignent les institutions cotées en Europe. L'Université catholique de Louvain possède ainsi son foyer américain, par lequel passe le P. Gerald Phelan au milieu des années vingt, ainsi que Mgr Fulton Sheen, évêque auxiliaire de New York. Le couvent d'études du Saulchoir voit passer, tout au long des années trente, son lot de dominicains canadiens, appelés à prendre plus tard des responsabilités au sein de l'ordre. Étienne Gilson attire quelques étudiants à l'EPHE : quelques dominicains canadiens, mais aussi Richard McKeon, par exemple, doyen du département de philosophie de l'Université de Chicago dans les années quarante, qui, après ses études à Columbia, était venu à Paris pour se former en philosophie médiévale. Maritain attire aussi quelques élèves à l'Institut catholique de Paris, dont l'un deviendra l'archevêque de Québec au moment du Concile, Mgr Maurice Roy:

«Je n'ai oublié, écrivait ce dernier en 1931, ni le salon de Meudon, ni les cours si vivants pendant lesquels vous nous initiiez avec tant de maîtrise à une méthode qui rajeunit les thèses de la philosophia perennis en les illustrant par les plus récentes expériences. De tout ce que je vous dois, comme disciple, j'ai voulu bien souvent vous remercier. (...) En fait, c'est avec émotion que je pense à tout ce que je vous dois. De vos cours à l'Institut catholique, du salon de Meudon, où madame Maritain nous accueillait avec une grâce si parfaite, je garde un incomparable souvenir ${ }^{34}$.

Au-delà de ces trajectoires personnelles, le cas le plus original est sans doute celui du PIMS de Toronto. Une des préoccupations majeures de l'Institut de Toronto fut en effet de promouvoir la formation de ses futurs professeurs, qui devaient être capables de couvrir le champ entier de la civilisation médiévale. Dans ce but, Gilson demande au Supérieur de Saint-Michael's College de lui confier une poignée de jeunes religieux prometteurs pour les envoyer suivre des études en Europe :

32. Lettre de J. Maritain au P. Phelan, 28 janvier 1945, SMCA.

33. Lettre de J. Maritain à François Mauriac, 10 mai 1948, Paris, Archives Doucet.

34. Lettre de l'abbé M. Roy à J. Maritain, 24 décembre 1931, AM. 
"Cela assurerait l'unité d'esprit et d'enseignement qui est nécessaire à une institution de ce type ; il en résulterait en outre un renforcement de l'ordre basilien, en lui donnant nombre de jeunes professeurs minutieusement entraînés dans leurs disciplines. (...) Telle que la situation nous apparaît maintenant du point de vue de nos besoins, nous conseillerions d'envoyer un de ces hommes à l'Université de Louvain, pour apprendre la philosophie de saint Thomas sous la direction de Mgr Noël ; un autre devrait aller à Rome, pour travailler la philosophie médiévale sous la direction du R.P. Théry, o.p. ; un troisième devrait aller à la Faculté Théologique de l'Université de Strasbourg, avec l'abbé Mollat, ou à Montpellier avec le Professeur Fliche, pour étudier l'histoire politique du Moyen Âge » ${ }^{35}$.

En 1932, au Supérieur des Basiliens qui lui demandait s'il ne valait pas mieux envoyer un religieux étudier la philosophie à l'Institut Catholique de Paris plutôt qu'à celui de Louvain, Gilson apporte une réponse qui témoigne de sa ferme volonté de puiser à la source de toutes les écoles d'Europe afin d'enraciner l'enseignement médiéval de Toronto dans la pluralité des approches européennes :

" Je n'ai rien contre les professeurs de philosophie thomiste de Paris, mais je crois sincèrement que Louvain est une meilleure place; le cursus général des études, malgré tous ses défauts, est mieux organisé qu'à l'Institut Catholique. Votre homme le trouvera certainement un peu sec, mais notre Institut prospérera de la diversité des influences, à supposer qu'il soit assez fort pour les assimiler. J'espère qu'il le sera. Mais n'envoyez qu'un seul homme à Louvain. J'ai vu le directeur de l'École des chartes à propos du P. Flahiff. Son opinion est que le P. Flahiff est non seulement un homme de valeur, mais aussi "très brillant" " 36 .

L'École des chartes est un lieu d'importance. Outre le P. Bernard Flahiff (1905-1989), basilien, qui enseigne à Toronto jusqu'à sa nomination au siège archiépiscopal de Winnipeg, en 1961, et qui est nommé cardinal par Paul VI, en 1969, elle forme en effet le P. Philip Moore, qui lance l'Institut d'Études médiévales sur le campus de Notre Dame. La correspondance du P. Moore avec le provincial de sa congrégation permet de comprendre comment un religieux du Midwest est arrivé à l'École des chartes :

" J'ai décidé de m'inscrire à l'École des chartes. Cette école est une des mieux organisées de Paris, et je n'hésite pas à dire à la fin de la première année qu'elle n'a nulle part d'égal pour la préparation dans tous les champs des études médiévales. En vérité, l'Institut catholique n'a rien ou pratiquement rien comme cours médiéval. On pourrait mentionner Maritain, mais il n'est pas en histoire, et ses livres sont bien meilleurs que son enseignement. (...) Le cas est différent avec Gilson, qui est à la fois un philosophe et un professeur inspirant. Mais Gilson était au Canada ${ }^{37}$.

La réponse inquiète du Provincial ne fut pas celle qu'imaginait le P. Moore, tout à la joie de sa première année à l'École des chartes à titre d'élève étranger. Ce n'est pas d'un historien, mais d'un philosophe dont la congrégation a besoin.

35. Lettre de Gilson au père McCorkell, 24 novembre 1929, SMCA.

36. Lettre d'É. Gilson au P. Carr, 7 mai 1932, SMCA.

37. Lettre du père Philip Moore au père James Burns, 11 juillet 1930, PAC. 
Le P. Moore est obligé de se justifier : «Si j’ai agi sottement, la raison est que j'ai pensé par erreur que je devais faire des études en histoire de la philosophie plutôt qu'en philosophie. Quand je disais vouloir devenir historien, j'avais à l'esprit historien de la philosophie médiévale " ${ }^{38}$. L'argument convainc et le P. Moore est autorisé à poursuivre ses études de chartiste.

Les chartistes américains ont ceci d'original par rapport à leurs homologues français qu'ils n'occupent pas des postes de conservateurs, mais enseignent les " études médiévales ». Les PP. Flahiff et Roy offrent, en outre, deux exemples de cardinaux nord-américains passés au moule du médiévisme dans des universités françaises durant les années trente.

\section{Pourquoi les Mediaeval Studies?}

Les raisons de l'intérêt pour le Moyen Âge sont multiples. L'universitaire américain honnête, qu'il fût littéraire, philosophe ou historien, ne pouvait pas se satisfaire de l'immense vide culturel qui séparait l'Antiquité de la Renaissance et qu'on lui avait soigneusement enseigné. Les témoignages sont éloquents : le cours de philosophie dispensé à Columbia dans les années vingt « sautait ainsi au-dessus de mille ans d'histoire, des philosophes hellénistiques de la Rome impériale à Descartes et à l'aube de la philosophie moderne, avec en passant la référence la plus brève à la pensée du Moyen Âge » ${ }^{39}$. La réputation du Moyen Âge était en outre en train de changer : plutôt que son obscurantisme, on commence à distinguer désormais sa "grande clarté »(Cohen, 1943). Avec Gilson, on prend également conscience de l'enracinement des premiers penseurs de la modernité européenne dans la tradition médiévale. Les Américains n'avaient de toute façon pas attendu cette réhabilitation historiographique et philosophique pour percevoir la beauté de l'art roman ou gothique... Outre ces raisons, suffisantes sans doute, il existe deux autres éléments, à la fois distincts et convergents, qui permettent, croyons-nous, de saisir les raisons plus profondes de l'intégration du médiévisme dans les structures académiques et culturelles américaines. Il y a une logique que l'on pourrait dire de " civilisation »; et une logique que l'on pourrait qualifier de religieuse. Dans les deux cas, la Première Guerre mondiale marque un tournant.

Le sauvetage culturel de la civilisation médiévale est l'un des enjeux revêtus par le médiévisme nord-américain. Les vétérans des guerres mondiales avaient pu voir de leurs propres yeux la montée de la barbarie en Europe, les incendies de bibliothèques, les autodafés, ou encore les pertes irrémédiables du patrimoine médiéval. En Europe, on se répète le mot de Valéry : "Nous autres, civilisations, nous savons que nous sommes mortelles ". Dans les discours parfois dramatisés

38. Lettre du père Moore au père Burns, 24 août 1930, PAC.

39. Mortimer Adler, Philosopher at Large. An Intellectual Autobiography, New York, 1977, pp. 81-83. 
des acteurs, on repère ainsi quelques analogies avec la fin de l'empire romain d'Orient. De même que les savants byzantins avaient fui l'ultime capitale de l'empire romain pour se réfugier à l'Ouest, de même les États-Unis accueillentils la culture médiévale avant qu'elle ne disparaisse tout à fait d'Europe. C'est en ces termes que Gilson défend la fondation de l'Institut de Toronto au début des années trente :

"La dernière guerre mondiale montre, par l'exemple de l'Allemagne, quel revers le savoir peut essuyer comme conséquence de tels conflits armés, et si ce qui arriva à l'Allemagne devait arriver à l'Europe entière, la seule espérance de sauver une tradition vieille de plusieurs siècles de l'assaut des barbares serait sa survie en Amérique " ${ }^{40}$.

Avec la Seconde Guerre mondiale, la menace d'une destruction par le feu devient de plus en plus plausible et ne manque pas ici ou là de se produire: l'exemple le plus tragiquement célèbre est sans doute l'incendie des archives royales de Naples en septembre $1943^{41}$. À l'été 1940, aussitôt après la chute de la France, l'American Council of Learned Societies et la Library of Congress, soutenus par la Fondation Rockefeller, mettent au point un ambitieux programme de reproduction des manuscrits du Moyen Âge et de la Renaissance des bibliothèques d'Angleterre et du Pays de Galles : sous les bombes, les photographes américains parviennent à reproduire dix mille codices du British Museum et des bibliothèques d'Oxford, de Cambridge et de quelques autres universités ${ }^{42}$. Les archivistes américains se mobilisent et lancent une campagne offensive de collecte des trésors médiévaux ${ }^{43}$.

Lors de son discours d'ouverture de l'année scolaire 1949, le P. Gerald Phelan résumait les sentiments américains d'alors : il revient à l'Université, disait-il, de " poursuivre les traditions culturelles de savoir qui jadis vitalisèrent l'Europe chrétienne, mais que l'Europe, maintenant épuisée à travers des conflits chroniques et des guerres amères, n'est plus capable de poursuivre malgré les efforts vaillants de quelques groupes de penseurs courageux " ${ }^{44}$. Le sentiment, répandu chez de nombreux Américains après les deux guerres mondiales, est qu'ils doivent prendre le relais culturel d'une Europe à l'agonie.

40. É. Gilson, "Aim and purpose », 2 pages manuscrites, années 1930 (sans date plus précise), SMCA.

41. Riccardo Filangieri, « Report on the destruction by the Germans, September 30, 1943, of the depository of priceless historical records of the Naples State Archives ", The American Archivist, 1944, pp. 252-254.

42. Eugene B. Power, "The Manuscript Copying Program in England ", The American Archivist, 1944, pp. 28-32 ; Lester K. Born, British Manuscripts Project: a Checklist of the Microfilms Prepared in England and Wales for the American Council of Learned Societies. 1941-1945, Washington, 1955 : l'avant-propos de cet inventaire indique 2652 bobines de microfilms, qui représentent cinq millions de pages de manuscrits.

43. Voir notamment Waldo Leland, "The Archivist in Times of Emergency ", The American Archivist, janvier 1941, pp. 1-12.

44. Discours de prise de charge du P. Phelan, 17 octobre 1949, 6 p., University of Notre Dame Archives, UDPL, 1/01. 
Aux États-Unis comme au Canada et en Europe, les études médiévales naissent également d'une critique de la modernité ${ }^{45}$. Les guerres, les crises de l'économie, les catastrophes du politique nourrissent une forme d'hostilité à la civilisation moderne, invitant certains à considérer le retour au Moyen Âge comme le détour nécessaire pour sortir de l'impasse d'une modernité fourvoyée. Dans son autobiographie La Nuit privée d'étoiles, Thomas Merton (1915-1968), alors étudiant à Columbia, oppose ainsi " la confusion et les souffrances du monde moderne " à « la simplicité profonde, candide et riche des XII ${ }^{\mathrm{e}}$ et XIII ${ }^{\mathrm{e}}$ siècles » (2004 : 139-146) qui commencent à l'attirer et le conduisent à l'abbaye cistercienne de Gethsémani (Kentucky).

Le programme d'enseignement de l'Institut de Notre Dame donne un exemple de ce recours aux études médiévales pour résoudre les déboires de l'aujourd'hui :

"Le monde souffre d'inanition en conséquence de la perte d'une tradition puissante et vivante. (...) Le but des études médiévales à Notre Dame est de re-posséder ce qui a été perdu, de faire nôtres, une nouvelle fois, les richesses intellectuelles des longs siècles de combat pour un sens métaphysique et spirituel. (...) Nous devons nous dévouer à la tâche de recouvrer le précieux dépôt de la vérité médiévale, parce que nous sentons que cette vérité peut restaurer et ordonner l'actuel désarroi intellectuel ${ }^{46}$.

En ces années trente où l'Amérique doute d'elle-même, l'Institut de Toronto a, pour Gilson, un rôle précis à jouer. L'institut «ne vise pas à ramener les hommes au Moyen Âge ", mais a pour but de rendre sens et raison à des notions que la modernité a dévaluées :

"Les seuls principes par lesquels une civilisation puisse vivre sont ceux par lesquels elle a été créée. Le christianisme a façonné ce que nous appelons aujourd'hui la culture occidentale. Les notions même de paix internationale, de démocratie, de justice sociale, d'amitié politique, des droits sacrés de la personne humaine, sont autant de conquêtes du christianisme contre la barbarie, dans laquelle nous sommes condamnés à retomber si nous ne sommes pas capables de tenir les vraies significations de ces principes et d'en produire, chaque fois que nécessaire, des justifications rationnelles $» 47$.

On le perçoit dans l'argumentation de Gilson: la logique religieuse est enchâssée dans la question de civilisation. Le Moyen Âge vaut surtout par son fort coefficient de catholicité et de christianisme : nul hasard si les universités catholiques s'approprient en conséquence les Mediaeval Studies. "L'Institut

45. Depuis la thèse de Gilson sur les origines médiévales de la pensée de Descartes (1913) et Antimoderne de Jacques Maritain (1922), une veine de l'intelligence française de l'entre-deuxguerres n'hésite pas à s'en prendre aux grands noms de la modernité - Descartes et Rousseau notamment -, et à fustiger les apports de la Renaissance. On peut évoquer sur ce point le livre de Nicolas Berdiaef, intitulé Un nouveau Moyen Âge, publié chez Plon dans la collection du «Roseau d'or» en 1927 : «L'humanisme n'a pas fortifié, il a débilité l'homme. (...) L'image même de l'homme est tout obscurcie. Et des esprits doués de quelque intuition remonteraient volontiers au Moyen Âge pour lui redemander les véritables origines de la vie humaine - pour lui redemander l'homme en un mot. »

46. Syllabus of the Graduate Courses in Mediaeval Studies, University of Notre Dame, 1934-1935, UNDA.

47. É. Gilson, "Aim and purpose », 2 pages manuscrites, années 1930 (sans date plus précise), SMCA. 
d'Études médiévales mérite tout notre soutien en tant qu'il offre une protection puissante contre toutes les invasions de la sécularisation ", écrivait l'archevêque de Toronto lors de l'inauguration de l'Institut ${ }^{48}$. C'est pour cette raison que les universités catholiques sont un maillon crucial pour saisir la diffusion des études médiévales en Amérique du Nord. Jusque dans les années soixante, identité catholique et culture médiévale sont intimement jointes. Ainsi le P. Moore de Notre Dame :

"Cela ne semble pas exagéré de dire que l'héritage intellectuel de l'Occident pourrait être peu à peu perdu aux Etats-Unis, à moins qu'il ne soit entretenu et défendu dans les écoles catholiques. On a besoin, en conséquence, d'une recherche vigoureuse sur les périodes patristiques et médiévales de notre passé. Pour la période médiévale, cela est non seulement vrai pour la théologie et la philosophie, mais aussi pour les autres branches du savoir. Dans une telle perspective, la signification et l'importance de notre Institut médiéval sont accentuées. Correctement développé, cela peut devenir une marque distinctive de Notre Dame en tant qu'université catholique ${ }^{49}$.

Au-delà des milieux catholiques nord-américains, la culture américaine est en pleine évolution, ce qui facilite l'acceptation et la diffusion des Mediaeval Studies. L'entre-deux-guerres, malgré quelques événements très étudiés comme le fameux "procès du singe » (1925), est marqué, chez les intellectuels, par une vague de sécularisation. John Dewey, au sommet de son influence, soulignait en 1926 la déchristianisation des intellectuels américains; il parlait ainsi du « déclin d'un intérêt aigu, voire d'une vraie croyance, dans le contenu de la révélation chrétienne » ${ }^{50}$. Au commencement de la Seconde Guerre mondiale, un ami américain de Jacques Maritain, écrivait dans le même sens que « le mot Dieu est curieux en anglais. Sur la page imprimée cela a l'air d'une faute d'impression " 51 . L'après-1945 sera au contraire marqué par un retour du religieux. Les études précises manquent sur cette évolution culturelle, qui tient également au contexte de Guerre froide : le religieux est alors réactivé dans la confrontation avec le communisme athée. Le dossier de la Partisan Review (New York, 1950) fournit un utile point de repère. Intitulé Religion and the Intellectuals, le dossier livre les contributions d'une trentaine d'auteurs, dont J. Dewey, J. Maritain, P. Tillich, H. Arendt, et met à jour les deux pôles, religieux et scientifique, des débats philosophiques au seuil des années cinquante. L'éditorial s'ouvre ainsi :

«Une des tendances les plus signifiantes de notre époque, et tout particulièrement de cette décennie, a été un nouvel intérêt envers la religion parmi les intellectuels et une défaveur croissante avec laquelle les perspectives séculières sont maintenant considérées dans un grand nombre de cercles qui prétendent au leadership culturel. Nul doute : le nombre d'intellectuels qui admettent des sympathies, des croyances ou des doctrines religieuses est plus fort aujourd'hui qu'il y a dix ou vingt ans. »

48. Sermon of the most Reverend McNeil, archbishop of Toronto delivered at St. Michael's College, September $30^{\text {th }} 1929$ at the opening of the Institute of Mediaeval Studies, p. 7.

49. P. Moore, Academic Development: University of Notre Dame, Past, Present and Future, 1960, dernière page.

50. John Dewey, in Zybura, 1926, pp. 29-31.

51. Lettre de G. Paulding à J. Maritain, 21 octobre 1943, Archives Maritain. 
L'éditorial poursuivait en qualifiant le commencement $\mathrm{du} \mathrm{xx}^{\mathrm{e}}$ siècle de "triomphe du naturalisme ", alors que l'on entend au milieu du siècle "une note insistante pour signaler que la civilisation occidentale ne peut pas survivre sans la réactivation des valeurs religieuses ". John Dewey, qui un quart de siècle plus tôt était prêt à enterrer le christianisme, confirme le diagnostic posé par la revue : il évoque cette fois «l'actuelle perte de foi en la science parmi les intellectuels. » La bonne réception du Moyen Âge chrétien est aussi fonction de ce revival religieux que connaissent les États-Unis au milieu du $\mathrm{XX}^{\mathrm{e}}$ siècle. La diffusion des Mediaeval Studies, concomitante d'un affaiblissement de "l'altérité catholique » (McCartin, 2003 : 7-29), ne relève donc pas de la seule culture catholique : la société américaine éduquée a pu aussi y chercher un Graal en réponse à un malaise ; elle y a quêté une «tradition » dans un monde en mutation.

Cela dit, la vague de sécularisation des décennies suivantes, dont la publication par le pasteur baptiste Harvey Cox de The Secular City, en 1965, fournit un commode repère ${ }^{52}$, oblige le médiévisme à s'ouvrir à des problématiques non religieuses. Avec sa plus large diffusion dans les milieux académiques ${ }^{53}$, on note, en même temps qu'une simplification orthographique - on parle désormais des "Medieval Studies"-, son élargissement thématique et géographique :

"Notre activité, écrit le président de la Mediaeval Academy pour le $50^{\mathrm{e}}$ anniversaire de la fondation, doit s'élargir dans toutes les directions, à tout l'univers médiéval. Nos yeux ont touché des lieux et des étoiles plus tard que d'autres: la loi médiévale, la technologie médiévale, l'économie médiévale, le monde slave, le monde musulman, pour donner quelques exemples, sont entrés dans notre champ d'études après un bon nombre d'années. Mais ce champ continue de s'étendre ${ }^{54}$.

Les études médiévales sont aussi renouvelées par les diverses modes universitaires. À l'histoire philosophique du Moyen Âge succède au fil des années une histoire plus politique, culturelle et populaire. L'Institut d'études médiévales de Montréal illustre les glissements successifs : «Il était peut-être inévitable que, dans un Québec qui se détachait toujours davantage de ses racines catholiques, l'intérêt pour la philosophie et la théologie du Moyen Âge finisse par s'émousser et perdre cette portée "existentielle" qui caractérisait la fondation initiale. Le projet collectif s'estompa peu à peu, chaque médiéviste préférant suivre l'optique spécifique de sa discipline " ${ }^{55}$. D'un autre témoin, le même constat : «Un brusque changement de cap se produisait à l'Institut d'Études médiévales de Montréal.

52. Traduction en française : La Cité séculière, essai théologique sur la sécularisation et l'urbanisation, Paris, Casterman, 1968.

53. Il faut ici évoquer les congrès de Kalamazoo dans le Michigan qui, tous les deux ans depuis le début des années soixante, rassemblent des centaines de médiévistes autour du Medieval Institute de la Western Michigan University et qui publient, depuis 1964, des Studies in Medieval Culture, ainsi que la revue Medieval Prosopography depuis le début des années quatre-vingts.

54. Stephen Kuttner, "A time for reflection ", Speculum, 1975, 50, p. 585.

55. Pierre Boglioni, "Sur les épaules des géants ", L’autre Forum, Montréal, septembre 2004, p. 31. 
Ce centre avait été au Québec, dans les décennies précédentes, le bastion de la recherche scientifique en philosophie médiévale. (...) Dans les années 1970 prévalent alors des approches plus sociologiques et plus littéraires, ainsi qu'en témoignent les thèmes retenus pour ses premiers colloques annuels : "la marginalité au Moyen Âge" en 1974, “l'érotisme au Moyen Âge" en 1976, "la culture populaire au Moyen Âge" en 1978 "(Pannaccio, 1998 : 146-149) ${ }^{56}$.

L'histoire de la religion populaire au Moyen Âge est elle-même suivie d'une historiographie rafraîchie à l'eau de jouvence de la Gender Theory. Au seuil des années quatre-vingt-dix, Speculum publie ainsi un ouvrage, Studying Medieval Women, Sex, Gender, Feminism (Partner, 1993), qui en bien des sens apparaît comme une re-visitation du titre même de la revue : le Speculum de 1925 était le miroir où le monde médiéval se reflétait ; le spéculum revendiqué en 1993 est l'instrument gynécologique contre lequel «consciemment ou inconsciemment » la revue s'était élevée en ses premiers temps pour promouvoir " la latinité, l'antimodernité et la masculinité » du Moyen Âge. Les auteurs soulignent l'impact du féminisme sur les Mediaeval Studies, avec une ouverture vers d'autres champs de la connaissance du Moyen Âge, et une extension du public touché par la culture médiévale : "Ce faisant, nous avons aussi revitalisé les études médiévales en général, attirant de nouveaux étudiants, invitant à de nouveaux travaux sur archives, provoquant de nouvelles discussions » (Bennett, 1993 : 7-29).

\section{Conclusion}

La question des Mediaeval Studies en Amérique du Nord est donc un enjeu à la fois archivistique, muséographique, académique, historique, philosophique, religieux, sociétal. Il faudrait pouvoir mettre ces aspects en parallèle avec d'autres domaines de connaissance où les transferts transatlantiques jouèrent également un rôle - dans l'évolution des sciences religieuses par exemple, en exégèse, avec notamment le développement des Biblical Studies.

En un sens, les Mediaeval Studies apparaissent comme un trait d'union culturel entre les deux continents et comme une reconnaissance de l'enracinement du Nouveau Monde dans la culture européenne médiévale. À la fin du XVIII siècle, Thomas Jefferson évoquait la «dette » culturelle des États-Unis : il n'y a pas d'indépendance de la culture pour l'auteur de la Déclaration d'Indépendance, qui désirait «faire quelque retour vers les nations plus anciennes, auprès desquelles nous sommes en dette ", et qui espérait que " dans cette tâche une part honorable serait assurée par l'université » ${ }^{57}$. Son vœu semble sur ce point comblé. 1972.

56. Voir aussi Pierre Boglioni et Benoît Lacroix, Les Religions populaires. Québec, PUL,

57. The Complete Jefferson, Saul K. Padover, (ed.), New York, 1943, p. 866. 
Mais le Moyen Âge au Nouveau Monde devient aussi, par le truchement des Mediaeval Studies, un nouveau Moyen Âge, renouvelé par la vie universitaire américaine. Dressées, pour partie, contre la sécularisation de la vie culturelle dans les années vingt, les Mediaeval Studies, sans perdre leur couleur d'origine, ont été, dans leurs grandes lignes, métamorphosées par le tournant séculier de la seconde moitié des années soixante, puis par celui des Cultural Studies qui ouvrent la discipline vers de nouveaux horizons. Le Moyen Âge ainsi théorisé, modernisé, esthétisé parfois, dématérialisé le plus souvent - la bobine de microfilm épuise-t-elle le manuscrit ? - est sans doute à peine moins loin du Moyen Âge que ne le sont Ivanhoé de Walter Scott ou les mediaeval fantasies des maîtres d'Oxford, Tolkien et Lewis. Mais échappe-t-on, au fond, à « l'invention du Moyen Âge » ? Peut-on interpréter le Moyen Âge sans recourir aux théories les plus actuelles, pour le meilleur ou pour le pire (Cantor, $1993: 38)^{58}$ ?

La question de la translatio des études médiévales en Amérique du Nord pose enfin la question de la translation du pouvoir en ce siècle de l'Empire américain. Qu'eût été l'empire de Charlemagne sans Alcuin, ni la renaissance carolingienne ? Le saint Empire américain devait chercher, pour élaborer une culture totale, universelle et impériale, à repousser le limes - " la Frontière » - de la chronologie et récapituler, en ses chaires, les siècles du Moyen Âge.

Florian MICHEL

Université de Paris XII

florian.michel77@free.fr

\section{Bibliographie}

BennetT Judith M., 1993, « Medievalism and Feminism », in Partner N.F., (dir.), Studying Medieval Women, Sex, Gender, Feminism, Cambridge, The Medieval Academy of America, pp. 7-29.

Berkhout Carl. T., 1982, "Medieval Research Centers in North America ", in Gentry F. G., Kleinheinz C., Medieval Studies in North America. Past, Present and Future, Kalamazoo, Medieval Institute Publications, pp. 97-120.

Boglioni Pierre, 2001, "Les traditions religieuses de l'Occident médiéval. Pensée chrétienne, religion populaire, hagiographie ", in Larouche J.-M., Ménard G., (dirs.), L'Étude de la Religion au Québec. Bilan et perspectives, Québec, PUL, pp. 195-196.

CANTOR Norman, 1993, Inventing the Middle Ages: the Lives, Works and Ideas of the great Medievalists of the Twentieth Century, New York, Harper Perennial.

Coffman George, 1926, "The Mediaeval Academy of America: historical background and prospect ", Speculum, 1, pp. 5-18.

58. Cantor citait en introduction (p. 18) une formule d'Umberto Eco: "Chacun a son idée - généralement corrompue - du Moyen Âge. » Cantor évoque le parallèle parfois tiré entre Abélard et Herbert Marcuse, tous deux adeptes du free love (p. 42). 
CoHen Gustave, 1945, "Progrès des études médiévales aux États-Unis ", Revue du Moyen Âge latin, Lyon, pp. 91-93.

-, 1942, Lettres aux Américains, Montréal, Éditions de l'Arbre.

-, 1943, La grande Clarté du Moyen Âge, New York, Édition de la Maison française.

Corbett James, Moore Philip, 1938, Petri Pictavensis allegoriae super tabernaculum Moysi, Publications in Mediaeval Studies, 3, UND.

Cusset François, 2003 [2005], French Theory : Foucault, Deleuze et Cie et les mutations de la vie intellectuelle aux États-Unis, Paris, La Découverte.

FoHLEN Claude, 1987, "Catholicisme américain et catholicisme européen : la convergence de "l'américanisme" ", Revue d'Histoire Moderne et Contemporaine, pp. 215-230.

Gentry Francis G., KLeInHeInZ Christopher, 1982, Medieval Studies in North America. Past, Present and Future, Kalamazoo, Medieval Institute Publications,

Haskins C. H., 1933, The Renaissance of the Twelth Century, Cambridge, Harvard University Press.

JoHn James, 1953, The College of Premontré in Mediaeval Paris, coll. " Texts and Studies in the History of Mediaeval Education ", Notre Dame, University of Notre Dame Press.

Libera Alain de, 1991, Penser au Moyen Âge, Paris, Seuil.

LOYER Emmanuelle, 2005, Paris à New York. Intellectuels et artistes français en exil 19401947, Paris, Grasset.

MARITAIN Jacques, 1944, "Philosophical cooperation and intellectual justice ", The Modern Schoolman, coll. "CEuvres complètes ", IX, pp. 271-300.

McAvoy Thomas, 1957 [1963], The Americanist Heresy in Roman Catholicism 18951900, Notre Dame, University of Notre Dame Press.

McCartin James, 2003, "The Waning of the "Catholic Other"... ", Revue Française d'Études Américaines, pp. 7-29.

Merton Thomas, [1951] 2004, La nuit privée d'étoiles, Paris, Albin Michel.

Michel Florian, 2007, "Le thomisme, un New Deal philosophique ? Jacques Maritain et la Bataille de Chicago (années 1930-années 1960) ", Recherches philosophiques, pp. 33-56.

-, 2010, La Pensée catholique en Amérique du Nord, réseaux intellectuels et échanges culturels entre l'Europe, le Canada et les États-Unis (années 1920-années 1960), Paris, Desclée deBrouwer.

Moore Philip, Academic Development: University of Notre Dame, Past, Present and Future, 1960.

-, 1936, The Works of Peter of Poitiers, Master in Theology and Chancellor of Paris (1193-1205), "Publications in Mediaeval Studies ", 1, Notre Dame, University of Notre Dame.

OwEns Joseph, 1988, « Mediaeval Studies fifty years later », Mediaeval Studies, pp. XI-XVI.

Panaccio Claude, 1998, "Philosophie médiévale ", in Klibansky R., Boulad-Ayoub J., (dirs.), La pensée philosophique d'expression française au Canada. Le rayonnement du Québec, Québec, Presses de l'Université Laval, pp. 145-162.

Partner Nancy F., (dir.), 1993, Studying Medieval Women, Sex, Gender, Feminism, Cambridge, The Medieval Academy of America.

Ricci Seymour de, Wilson W., Census of Medieval and Renaissance Manuscripts in the United States and Canada, 3 vol., New York, 1935-1940.

Rorimer James, 1938 [1963], The Cloisters, New York, 1938.

SHOoK Laurence, 1984, Etienne Gilson, Toronto, PIMS. 
Van SteEnberghen F., 1951, « Les Études médiévales en Amérique », Revue philosophique de Louvain, pp. 411-412.

-, 1994, "Aux origines de l'Institut d'Études Médiévales de Louvain-La-Neuve ", in Follon J., McEvoy A. J., (dirs.), Actualité de la pensée médiévale. Recueil d'articles, Louvain-la-Neuve, Peeters, pp. 17-21.

Willard James, 1926, Progress of Mediaeval Studies in the United-States of America, Boulder, Colorado.

Zybura J.S., 1926, Present Day Thinkers and the New Scholasticism, New York, Herder Book.

\section{Résumé}

Les études médiévales en Amérique du Nord se structurent au milieu des années vingt et suscitent un échange culturel entre le "Nouveau Monde " et l'Europe: nombreux sont les professeurs, étudiants ou manuscrits qui circulent alors entre les deux rives de l'océan. Quels sont les vecteurs de cet échange? Quelles en sont les formes et les causes? Quelles sont les métamorphoses des "mediaeval studies " en Amérique du Nord? Appuyé sur des archives situées aux États-Unis, au Canada et en France, cet article essaie de répondre à ces questions d'bistoire culturelle, dont le fort coefficient religieux est ici mis en évidence.

Mots-clés : études médiévales, Amérique du Nord, archives, échange culturel.

\section{Abstract}

"Mediaeval Studies" originated as an academic discipline at North American universities in the 1920s. This new field prompted a cultural exchange between the "New World" and Europe: numerous professors, students, and manuscripts circulated across the Atlantic. What were the vehicles for this exchange? What were its causes? What forms did this exchange take? How did "Mediaeval Studies" evolve in North America? Drawing on archives in the United States, Canada, and France, this article responds to these questions and reveals the importance of religion in understanding the development of "Mediaeval Studies".

Key words: mediaeval studies, North America, archives, cultural exchange.

\section{Resumen}

Los Estudios Mediales en Norteamérica se estructuraron durante los años 20 y suscitaron un intercambio cultural entre el "Nuevo Mundo" y Europa: numerosos profesores, estudiantes y manuscritos circularon por las dos riberas del océano. ¿Cuáles fueron los vectores de este intercambio? ¿Cuáles fueron las causas y los modos? ¿Cómo se metamorfosearon y evolucionaron los "Estudios Medievales" en Norteamérica? Apoyándonos en archivos de Estados Unidos, Canadá y Francia, este artículo responde a estas preguntas sobre la historia cultural y muestra la importancia de la religión para la comprensión del desarrollo de los "Estudios Medievales".

Palabras clave: estudios medievales, America del Norte, archivo, intercambios culturales. 